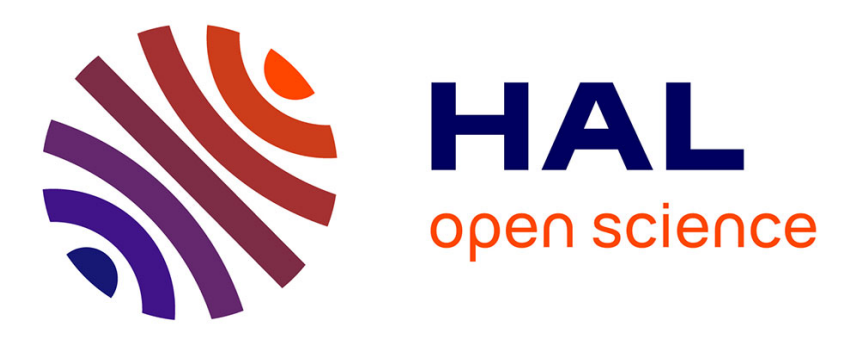

\title{
Optimal time control to swing-up the inverted pendulum-cart in open-loop form
}

\author{
Abdelkader Merakeb, Farida Achemine, Frédéric Messine
}

\section{To cite this version:}

Abdelkader Merakeb, Farida Achemine, Frédéric Messine. Optimal time control to swing-up the inverted pendulum-cart in open-loop form. 11th IEEE International Workshop ofElectronics, Control, Measurement, Signals and their application to Mechatronics (ECMSM 2013), Jun 2013, Toulouse, France. pp.1-4, 10.1109/ECMSM.2013.6648967 . hal-01178575

\section{HAL Id: hal-01178575 \\ https://hal.science/hal-01178575}

Submitted on $20 \mathrm{Jul} 2015$

HAL is a multi-disciplinary open access archive for the deposit and dissemination of scientific research documents, whether they are published or not. The documents may come from teaching and research institutions in France or abroad, or from public or private research centers.
L'archive ouverte pluridisciplinaire HAL, est destinée au dépôt et à la diffusion de documents scientifiques de niveau recherche, publiés ou non, émanant des établissements d'enseignement et de recherche français ou étrangers, des laboratoires publics ou privés. 


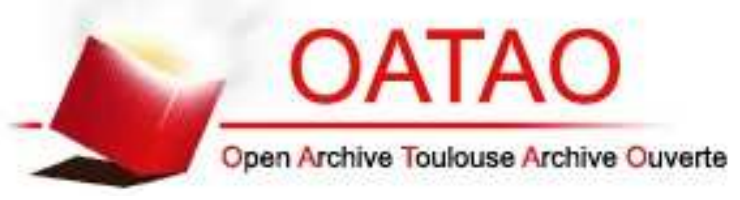

\section{Open Archive TOULOUSE Archive Ouverte (OATAO)}

OATAO is an open access repository that collects the work of Toulouse researchers and makes it freely available over the web where possible.

This is an author-deposited version published in : http://oatao.univ-toulouse.fr/ Eprints ID : 13011

URL: http://dx.doi.org/10.1109/ECMSM.2013.6648967

To cite this version : Merakeb, Abdelkader and Achemine, Farida and Messine, Frédéric Optimal time control to swing-up the inverted pendulum-cart in open-loop form. (2013) In: Electronics, Control, Measurement, Signals and their application to Mechatronics (ECMSM 2013), 24 June 2013 - 26 June 2013 (Toulouse, France).

Any correspondance concerning this service should be sent to the repository administrator: staff-oatao@listes-diff.inp-toulouse.fr 


\section{Optimal Time Control to Swing-Up the Inverted Pendulum-Cart in Open-Loop Form}

\author{
Abdelkader Merakeb \\ and Farida Achemine \\ Département de Mathématiques, Université Mouloud \\ Mammeri de Tizi-Ouzou, 15000 Algérie. \\ Email: merakeb_kader@yahoo.fr \\ acheminef@yahoo.fr
}

\begin{abstract}
This work deals with simulation on an Inverted Pendulum (IP). The control strategy of an IP is split into two main control phases: (i) swing-up control to bring back the pendulum from the downward position to the upward one, and (ii) upright stabilization control to maintain the pendulum to the upright vertical position. In the case (ii), a feedback or a neuro-fuzzy controller is used to stabilize the pendulum cart, while in the first case (i), a non-linear controller based on the energy of the pendulum is used in order to reach the desired performance with a minimum number of swings. Our contribution is to present a simulation using MatLab of time-optimal control system for swinging-up the pendulum, with a single control law in an open-loop form. From the bang-bang structure of the timeoptimal control resulting from the necessary condition of the Pontryagin Maximum Principle, the solution obtained from direct discretization method is adjusted by using Newton based method.
\end{abstract}

Keywords-Inverted Pendulum; Swing-up control; Time optimal control; Bang-Bang structure; Unstable equilibrium.

\section{INTRODUCTION}

The control of an Inverted Pendulum (IP) is one of the most important classical problems in Engineering command, and resembles the control systems that exist in robotic arms. IP has two equilibrium points, one of which is stable while the other is unstable. The stable equilibrium point corresponds to a state in which the pendulum is pointing downward. In the absence of any control force, the system will naturally return to this state. The unstable equilibrium point corresponds to a state in which the pendulum raises strictly upward and thus, requires a control force to reach and maintain this position.

Rigid Broom Balancing (Inverted Pendulum on a cart) is the problem that involves a cart equipped with a motor that drives it along an horizontal track able to move backward and forward, and a pendulum hinged to the cart at the bottom of its length such that the pendulum can move in the same plane as the cart. The user is able to manipulate the position and the velocity of the cart through the motor and the track restricts the cart to move in the horizontal direction. The goal is to stabilize the IP such that its the position on the track is controlled quickly and accurately such that the pendulum is always erected in its inverted position during such movements. Different control schemes were discussed to swing the pendulum from the downward position to the upright one, as described in [1], [4], [6]. The users are not

\author{
Frédéric Messine \\ ENSEEIHT-IRIT, UMR-CNRS 5055, \\ 2 rue Camichel, 31000 Toulouse, France. \\ Email: Frederic.Messine@n7.fr
}

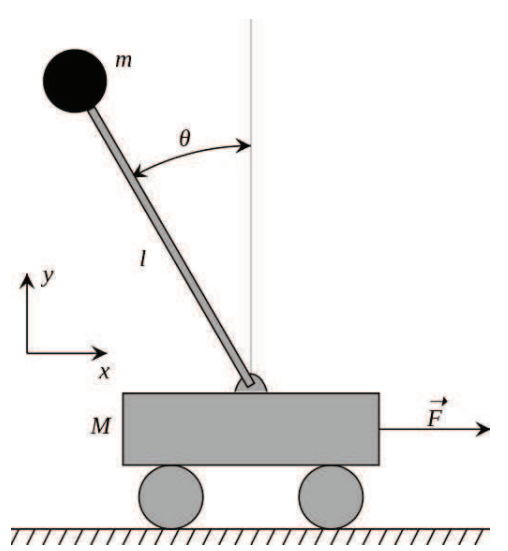

Fig. 1. Pendulum phenomenological model.

interested so much in the time to swing-up the pendulum while their objective is its stabilization when it reaches its unstable equilibrium point. This paper describes a method to swingup a pendulum attached to a cart in a minimum time with a control in open-loop form. The necessary condition derives from the Pontryagin Maximum Principle and yields that the optimal time control has a bang-bang structure. Unfortunately, the use of shooting method based on the indirect method does not give any satisfactory results because of the hardness of the non-linear dynamics of the system. We focus then on the discretization method to provide us an approached solution. This last solution will be used to be refined via a finishing procedure by taking into account the structure of the control.

In Section 2, the differential equations which come from the IP system are presented yielding the optimal time control problem that we have to solve. In section 3, we deduce the structure of the optimal time control by applying the necessary condition of the PMP. By discretizing the optimal control problem, we propose in Section 4 a method to refine the solution obtained, and some numerical simulations are performed. In Section 5, we conclude.

\section{InVERTED PENDUlum MODEL}

Mathematical models of mechanical systems are usually described by Hamiltonian or Euler-Lagrange equations. 
In figure 1, a model of the IP systems is displayed. The model used in this paper consists in two rods symmetrically hinged to a cart which can move horizontally by means of horizontal forces, driven by DC motor which is the control of the system. The cart is able to move on a limited horizontal rail with length $\left|L_{r}\right| \leq 0.5 \mathrm{~m}$, and the force of the DC motor on the cart is denoted by $F$. The system has one input and four outputs. The outputs are: (i) the cart horizontal distance $x$ in relation to the center of the track; (ii) the angular poles $\theta$ from the downward equilibrium point; (iii) cart velocity $\dot{x}$ and (iv) pendulum angular velocity $\dot{\theta}$. The (control) input designated by $u$ is the voltage $V_{m}$ that drives the DC motor.

Two points have to be kept in mind when the controllers are designed. The cart position and the control signal are both bounded in a real time application. The bound for the control signal is set to $[-25 \mathrm{~V}, 25 \mathrm{~V}]$ and the cart position is physically bounded by the rail length in the interval $[-0.5 m, 0.5 m]$. We consider in this model that $\theta=0$ is the stable position (the pendulum is below) and $\theta= \pm \pi$ is the unstable position (the pendulum is on the top).

By applying the law of the dynamic on the inverted pendulum cart system, we get the following non-linear equations of motion:

$$
\begin{gathered}
(m+M) \ddot{x}+b \dot{x}-m l \cos (\theta) \ddot{\theta}+m l \sin (\theta) \dot{\theta}^{2}=F, \\
-m l \ddot{x} \cos (\theta)+N \ddot{\theta}+d \dot{\theta}+m g l \sin (\theta)=0 .
\end{gathered}
$$

The system has several physical parameters associated with its components that are required for determining the differential equations. They are described in Table II. The values of these parameters are pulled from [1].

TABLE I. Pendulum Parameters.

\begin{tabular}{|c|l|l|}
\hline Parameter & Significance & Value \\
\hline$K_{m}$ & DC motor torque constant & $0.05 \mathrm{Nm}$ \\
$K_{b}$ & Gearbox Gearing ratio & $0.05 \mathrm{~N} / \mathrm{A}$ \\
$R$ & Motor Armature Resistance & $2.5 \Omega$ \\
$r$ & Motor pinion radius & $0.0148 \mathrm{~m}$ \\
$M$ & Cart mass & $2.4 \mathrm{~kg}$ \\
$m$ & Pendulum mass & $0.23 \mathrm{~kg}$ \\
$g$ & Gravity & $9.81 \mathrm{~m} / \mathrm{s}^{2}$ \\
$l$ & Pole length & $0.36 \mathrm{~m}$ \\
$N$ & Moment of Inertia of the pole & $0.099 \mathrm{~kg} \cdot \mathrm{m}^{2}$ \\
$b$ & Cart friction coefficient & $0.05 \mathrm{Ns} / \mathrm{rad}$ \\
$d$ & Pendulum damping coefficient & $0.005 \mathrm{Nms} / \mathrm{rad}$ \\
\hline
\end{tabular}

The force that the DC motor provides on the cart is dependent on the input voltage $u$ and on the velocity of the cart $\dot{x}$. The relationship is given by:

$$
F=\frac{K_{m} K_{b}}{R r} u-\frac{K_{m}^{2} K_{b}^{2}}{R r^{2}} \dot{x} .
$$

The state vector of the inverted pendulum-cart system is $X=\left(x_{1}, x_{2}, x_{3}, x_{4}\right)^{t}=(x, \dot{x}, \theta, \dot{\theta})^{t}$, where $x_{1}=x$ is the cart position, $x_{3}=\theta$ represents the pendulum angle (see Fig. 1), and $x_{2}=\frac{d x}{d t}, x_{4}=\frac{d \theta}{d t}$ are the velocity and the angular velocity of the cart and the pendulum, respectively. From equations (1), (2) and (3), the dynamic equations of the inverted pendulum-cart system are given by:

$$
\dot{X}=f(X)+b(X) u \Leftrightarrow\left\{\begin{array}{l}
\dot{x}_{1}=x_{2} \\
\dot{x}_{2}=f_{1}(X)+b_{1}(X) u \\
\dot{x}_{3}=x_{4} \\
\dot{x}_{4}=f_{2}(X)+b_{2}(X) u
\end{array}\right.
$$

where

$$
\left\{\begin{array}{l}
b_{1}(X)=\frac{N K_{m}}{R r} h_{1}(X) \\
b_{2}(X)=\frac{l m K_{m} \cos (\theta)}{R r} h_{1}(X) \\
f_{1}(X)=h_{1}(X)\left[h_{2}(X)+h_{3}(X)+h_{4}(X)\right] \\
f_{2}(X)=h_{1}(X)\left[h_{5}(X)+h_{6}(X)\right]
\end{array}\right.
$$

and

$$
\left\{\begin{array}{l}
h_{1}(X)=\left(N(M+m)-l^{2} m^{2} \cos ^{2}(\theta)\right)^{-1} \\
h_{2}(X)=-\left(\frac{N K_{b} K_{m}}{R r^{2}}+N b\right) \dot{x} \\
h_{3}(X)=-g l^{2} m^{2} \cos (\theta) \sin (\theta) \\
h_{4}(X)=-d l m \dot{\theta} \cos (\theta)-N l m \dot{\theta}^{2} \sin (\theta) \\
h_{5}(X)=-\left(\frac{l m K_{b} K_{m}}{R r^{2}}+b l m\right) \dot{x} \cos (\theta)-d h \dot{\theta} \\
h_{6}(X)=-g(M+m) l m \sin (\theta)-l^{2} m^{2} \dot{\theta}^{2} \cos (\theta) \sin (\theta)
\end{array}\right.
$$

The problem that we are interested in, can be formulated as an optimal time control problem in presence of state constraint on $x_{1}(t)$. We have to solve:

$$
\left\{\begin{array}{l}
\min _{X(t), u(t)} J\left(t_{f}, X, u\right)=\min \int_{0}^{t_{f}} d t \\
\text { s.t. } \quad \dot{X}=f(X)+b(X) u \\
\quad\left|x_{1}(t)\right| \leq 0.5 \\
|u(t)| \leq 25 \\
\left(x_{1}(0), x_{2}(0), x_{3}(0), x_{4}(0)\right)=\left(\begin{array}{ll}
0,0,0,0 & 0
\end{array}\right) \\
\left(x_{2}\left(t_{f}\right), x_{3}\left(t_{f}\right), x_{4}\left(t_{f}\right)\right)=(0, \pm \pi, 0)
\end{array}\right.
$$

\section{THE USED SWING-UP CONTROLLER DESIGN}

Different control schemes were discussed to swing the pendulum from the downwards position to the upright position. One of these methods, namely heuristic controller, provides a constant voltage in the appropriate direction, and drives the cart along the track repeatedly. Thus, it will repeat this action until the pendulum is close enough to the upright position such that the stabilizing controller can be triggered to maintain this balanced state [1], [4].

Another scheme is an energy controller that regulates the amount of energy in the pendulum. This controller inputs energy into the cart-pendulum system until it attains the energy state that corresponds to the pendulum in the upright position. Similarly to the heuristic control method, the energy control method will also switch to the stabilizing controller when the pendulum is close to the upright position.

The switch that triggers the stabilizing controller in both cases is activated when the pendulum is within 0.1 radians of the upright position and the angular velocity is slower than 2.5 radians per second [2], [6], [8].

Although the swing-up controllers described above are on the feedback form, the objective in this work is to swing-up the pendulum from the downward position $\theta=0$ to the upright one $\theta= \pm \pi$ in minimum time with an open-loop form.

\section{INDIRECT METHOD USING PMP}

In this case, we consider the Problem (4) and we relaxe the state constraint $\left|x_{1}(t)\right| \leq \infty$. One can understand that we can solve the relaxed problem as considering the rail is long enough, but this constraint could be restored after resolving the problem by replacing the cart at the beginning in the adequate 
position in order to satisfy this constraint. From the PMP, we deduce the expression of the optimal time control of Problem (4), which has a bang-bang structure. Indeed, we introduce the adjoint states variables $p(t)=\left(p_{1}(t), p_{2}(t), p_{3}(t), p_{4}(t)\right)$, and we define the Hamiltonian function as:

$H(t, X, p, u)=p_{0}+p_{1} x_{2}+p_{2}\left(f_{1}(X)+b_{1}(X) u\right)+p_{3} x_{4}+$ $p_{4}\left(f_{2}(X)+b_{2}(X) u\right)$,

which is linear in relation with $u$. With $p_{0}=-1$ (maximum principle), the adjoint states variables verify the equations $\dot{p}=$ $-\frac{\partial H(t, X, p, u)}{\partial X}$ and are given by

$$
\left\{\begin{aligned}
\dot{p}_{1}=-\frac{\partial H}{\partial x_{1}}= & 0 \\
\dot{p}_{2}=-\frac{\partial H}{\partial x_{2}}= & -p_{1}-p_{2}\left(\frac{\partial f_{1}(X)}{\partial x_{2}}+\frac{\partial b_{1}(X)}{\partial x_{2}} u\right) \\
& -p_{4}\left(\frac{\partial f_{2}(X)}{\partial x_{2}}+\frac{\partial b_{2}(X)}{\partial x_{2}} u\right) \\
\dot{p}_{3}=-\frac{\partial H}{\partial x_{3}}= & -p_{2}\left(\frac{\partial f_{1}(X)}{\partial x_{3}}+\frac{\partial b_{1}(X)}{\partial x_{3}} u\right) \\
& -p_{4}\left(\frac{\partial f_{2}(X)}{\partial x_{3}}+\frac{\partial b_{2}(X)}{\partial x_{3}} u\right) \\
\dot{p}_{4}=-\frac{\partial H}{\partial x_{4}}= & -p_{2}\left(\frac{\partial f_{1}(X)}{\partial x_{4}}+\frac{\partial b_{1}(X)}{\partial x_{4}} u\right)-p_{3} \\
& -p_{4}\left(\frac{\partial f_{2}(X)}{\partial x_{4}}+\frac{\partial b_{2}(X)}{\partial x_{4}} u\right)
\end{aligned}\right.
$$

The maximum of the Hamiltonian $H$ leads to the optimal control which has a bang-bang structure:

$$
u(t)=V_{\max } \operatorname{sign}\left(p_{2} b_{1}(X)+p_{4} b_{2}(X)\right)
$$

where $V_{\max }=25 \mathrm{~V}$ is the maximum voltage delivered by the DC motor.

The classical approach based on the PMP (indirect method), known for its speed and accuracy, has been tested on this optimization problem. However, its implementation using shooting techniques may (in practice) deal with some difficulties. Indeed, the shooting method consists to find a zero of the shooting function $S(y)=\hat{z}\left(t_{f}\right)-(0, \pm \pi, 0)$, where $\hat{z}$ corresponds to the integration of the Initial Value Problem (IVP) associated with the original problem

$$
(I V P)\left\{\begin{array}{c}
\dot{X}=f(X)+b(X) u \\
\dot{p}=-\frac{\partial H(t, X, p, u)}{\partial X} \\
X(0)=0 \\
p(0)=y .
\end{array}\right.
$$

After applying necessary conditions of optimality, this method has to solve a system of non-linear differential equations. However, its main drawback is the need of a correct starting point: as this method typically involve applying a quasiNewton based algorithm using the shooting function, the radius of convergence can be very low, depending on the regularity of the problem. The shooting method does not converge systematically, and one understands that it is not realistic to hope to solve the relaxed Problem (4) by this way.

\section{Direct METHOd USING DISCRETIZATION TECHNIQUES}

Considering direct methods which traditionally involve total or partial discretizations of the problem, we have to use various local approaches to solve the arising optimization problem. These methods are generally imprecise and they can lead to the resolution of large scale problems depending on the used step of discretization.

At the heart of a well-founded discretization method for solving optimal control problems, one has the following three
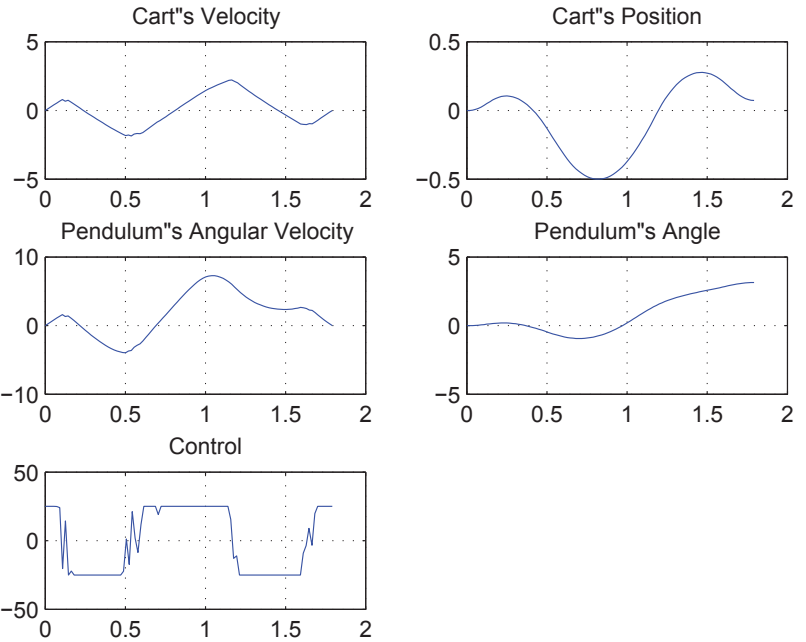

Fig. 2. Simulation of Swing-up control in optimal time.

fundamental components: - methods for solving the differential equations and integrating functions; - a method for solving a system of non-linear algebraic equations ; and - a method for solving a non-linear optimization problem.

Methods for solving differential equations and integrating functions are required for all numerical methods in optimal control. In a direct method, the numerical solution of differential equations is combined with non-linear optimization. In this work, the time-marching is the considered approach used for solving the differential equations with an explicit fourth order Runge-Kutta integrator.

In a direct method, the state and the control are discretized in some manner and the problem is transcribed to a nonlinear programming problem (NLP). The NLP is then solved using well-known optimization techniques (we use the fmincon MatLab subroutine). In a direct method, the optimal solution is found by transcribing an infinite-dimensional optimization problem into a finite-dimensional one. In Figure 2, the obtained solution is drawn, and one remarks that the optimal time control is not a bang-bang control but possesses a structure close to a switching function. This structure will be exploited from the approximated switching times and a finishing procedure is implemented. Note that the corresponding minimum time is about 1.79 seconds.

\section{A. Finishing procedure}

A system of non-linear algebraic equations can be considered equivalently to root-finding one. In this case, where all of the algebraic equations can be written as equalities, we have a problem of the form $S(y)=0$. The most common method for solving a multidimensional root-finding problem is a Newton based method, and an initial point is made of the vector $y$. It is well-known that the Newton method converges when the initial point is close to a root.

The finishing procedure consists in constructing the solution of the system of non-linear equations. Then the system 

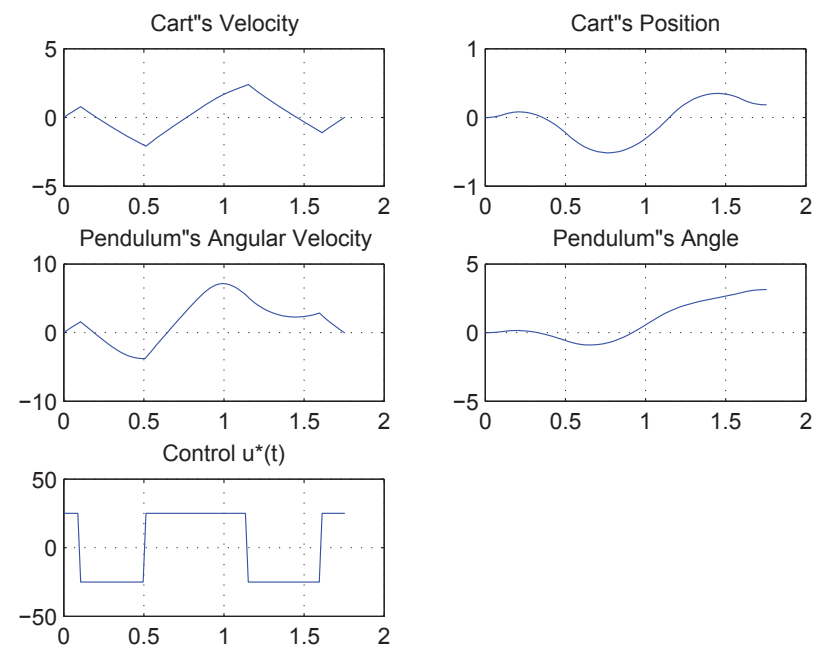

Fig. 3. Solution adjusted by the finishing procedure.

is solved using a Newton based method with the initial approximation which is uniquely determined from the switch times of the problem obtained at the end of the algorithm which solve a non-linear optimization problem (7). According to Newton based method, the switching function (bang-bang control) is constructed using the fsolve MatLab subroutine. The optimal time control $u^{*}(t)$ corresponding to the switching function is drawn in Figure 3 and the minimum time is about 1.75 seconds.

\section{CONCLUSION}

This study presents the simulation and the results of an optimal time control problem which deals with the balancing of a cart inverted pendulum driven by a motor. By using tools from optimal control theory through the PMP, we have obtained a description of the optimal time control problem, for the problem with the voltage of the DC motor as control inputs. In order to determine the solution, we used the direct method based on the discretization techniques and the computations are performed using fmincon from MatLab. From the bangbang structure of the optimal control, the finishing procedure based on the switching times is used. It operates with a Newton based method. This approach appears to work efficiently very well for the resolution of the system studied here. Further study on this system would bring significant benefits to robotic and control professional.

\section{REFERENCES}

[1] R. Mellah, F. Lahouazi, S. Djennoune, S. Guermah and R. Toumi, Composite Sliding Mode Control of Inverted Pendulum, International Journal of Control, Automation and Systems, Vol. 1, No. 3, May 2012.

[2] W. Zhong and H. Rock, Energy and Passivity Based Control of the Double Inverted Pendulum on a Cart, Proceedings of the 2001 IEEE International Conference on Control Applications, September 5-7, Mexico City, 2001.

[3] A. Bogdanov, Optimal Control of a Double Inverted Pendulum on the Cart, Technical Report CSE-04-006, OGI School of Science and Engineering, OHSU, 2004
[4] M. Bugeja, Non-Linear Swing-Up and Stabilizing Control of an Inverted Pendulum system, University of Malta, Msida, Malta, 2002.

[5] C. A. Ibanez, O. Gutierrez Frias and M. Suarez Castanon, LyapunovBased Controller for the Inverted Pendulum Cart System, Nonlinear Dynamics 40: 367374, Springer 2005.

[6] K. J. Astrom and K. Furuta, Swinging Up a Pendulum by Energy Control, Automatica, vol. 36, no. 2, pp. 287-295, February 2000.

[7] W. Zhong, Yang Chen and Fang, Minimum-Time Swing-up of a Rotary Inverted Pendulum by Iterative Impulsive Control, Proceeding of the 2004 American Control Conference, June 30-July 2, Boston, pp:1335-1340, 2004.

[8] N. Muskinja and B. Tovornik, Swinging Up and Stabilization of a Real Inverted Pendulum, IEEE Transactions on Industrial Electronics, Vol. 53, No. 2, pp:631-639, 2006.

[9] F.L. Chernousko, S.A. Reshmin, Time-optimal swing-up feedback control of a pendulum, Nonlinear Dyn., Vol 47, pp. 65-73, 2007.

[10] P. Mason, M. Broucke and B. Piccoli, Time Optimal Swing-Up of the Planar Pendulum, IEEE Transactions on Automatic Control, Vol. 53, No. 8, pp. 1876-1886, 2008.

[11] P. Melba Mary and N. S. Marimuthu, Minimum Time Swing Up and Stabilization of Rotary Inverted Pendulum Using Pulse Step Control, Iranian Journal of Fuzzy Systems Vol. 6, No. 3, pp. 1-15, 2009.

[12] V. Sukontanakarn and M. Parnichkun, Real-Time Optimal Control for Rotary Inverted Pendulum, American Journal of Applied Sciences 6 (6): 1106-1115, 2009. 